\title{
An insect eye based image sensor with very large field of view
}

\author{
Els Moens ${ }^{*} a$, Youri Meuret ${ }^{a}$, Heidi Ottevaere ${ }^{a}$, Mukul Sarkar $^{b}$, David San Segundo Bello $^{c}$, \\ Patrick Merken ${ }^{d}$, Hugo Thienpont ${ }^{a}$ \\ ${ }^{a}$ Vrije Universiteit Brussel, Faculty of Engineering, Brussels Photonics Team B-PHOT, TONA \\ FirW, Pleinlaan 2, B-1050 Brussel, Belgium; \\ ${ }^{b}$ IMEC / TU Delft, High Tech Campus 31, 5656 AE Eindhoven, Netherlands; \\ ${ }^{c}$ IMEC, Kapeldreef 75, 3001 Leuven, Belgium; \\ ${ }^{d}$ Royal Militairy Academy, Renaissancelaan 30, 1000 Brussel, Belgium;
}

\begin{abstract}
In this paper we discuss the design of a novel miniaturized image sensor based on the working principle of insect facet eyes. The main goals are to design an imaging system which captures a large field of view (FOV) and to find a good trade-off between image resolution and sensitivity. To capture a total FOV of $124^{\circ}$, we split up this FOV into 25 different zones. Each of these angular zones is imaged by an isolated optical channel on our image sensor. There is an overlap between the zones to cover the full FOV but the different zones are imaged on separated regions at the image sensor. Every optical channel in the designed component consists of two lenses that are tilted with respect to each other and the optical axis. Because of this tilt of the lenses, we are able to minimize field curvature and distortion in the obtained images at the detector, and have an angular resolution below $1^{\circ}$. The optical system was implemented and optimized in the ray-tracing program ASAP. The parameters (in one channel) that are optimized to obtain this large FOV with a good image resolution and sensitivity are the radius of curvature of the two lenses, their conical factor and their tilt in two directions with respect to the optical axis of the complete system. The lenses are each placed on a pedestal that connects the lens to a planar substrate. We also add absorbing tubes that connect the two lenses in one channel to eliminate stray-light between different optical channels. The obtained image quality of the design is analyzed using our simulation model. This is determined by different parameters as there are: modulation transfer function, distortion, sensitivity, angular resolution, energy distribution in each channel and channel overlap. The modulation transfer function shows us that maximum contrast in the image is reached up to $0.3 \mathrm{LP} /{ }^{\circ}$, distortion is maximal $21 \%$ in one of the 25 different channels, the sensitivity is $0.3 \%$ and the resolution is better than $1^{\circ}$.
\end{abstract}

Keywords: Micro-optics, optical design, wide field of view, image quality, image sensor

\section{INTRODUCTION}

Today lots of applications like endoscopy ${ }^{1,2}$ and security cameras require wide field of view (FOV) image sensors. ${ }^{3}$ In this paper we discuss the design of a new wide FOV $\left(124^{\circ}\right)$ micro-optical imaging system. Except for the wide FOV, it is our goal to design an image sensor with a good trade-of between obtained image quality and sensitivity.

For designing the micro-optical system, we were inspired by nature. Indeed our design is based on nature's insect facet eyes, which also have a very large FOV. Each facet eye is built up out of hundreds to thousands channels, called ommatidia. ${ }^{4}$ These capture light, with certain angles of incidence, from the outside world and guide it to the receptor cells. Since these ommatidia are placed on a curved surface these insect facet eyes have a very large FOV. Two types of facet eyes exist: superposition and apposition eyes (Fig. 1). In superposition eyes, each receptor captures light from several neigboring channels. In apposition eyes, each receptor only receives light from a single channel. The main difference between these two types of facet eyes is the sensitivity which is higher in the superposition eyes. On the other hand, in apposition eyes, the resolution is better. Light that enters a single channel in an apposition eye is guided through the ommatidium and on the receptor cells the light is captured. The angle between the different ommatidia axes is almost equal to the acceptance angle of a

\footnotetext{
* emoens@vub.ac.be;phone +32 (0)2 62913 81; fax +32 (2) 62934 50; www.b-phot.org
} 
single ommatidium. Since the FOV of one channel abuts the field of its neighbor, an overal erect image made up of a mosaic of adjacent FOVs is produced. In superposition eyes the receptor cell is a single sheet, not a combination of several cells in each ommatidium. ${ }^{5}$ Each optical part in the ommatidia of the superposition eye redirects a parallel beam of light to form another parallel beam on the same side of the optical axis. There are three possible ways to do this in superposition eyes: a two-lens telescope, a plane mirror and a combination of a curved mirror and a lens. ${ }^{4}$ The images from different ommatidia are superimposed to form a single erect image.
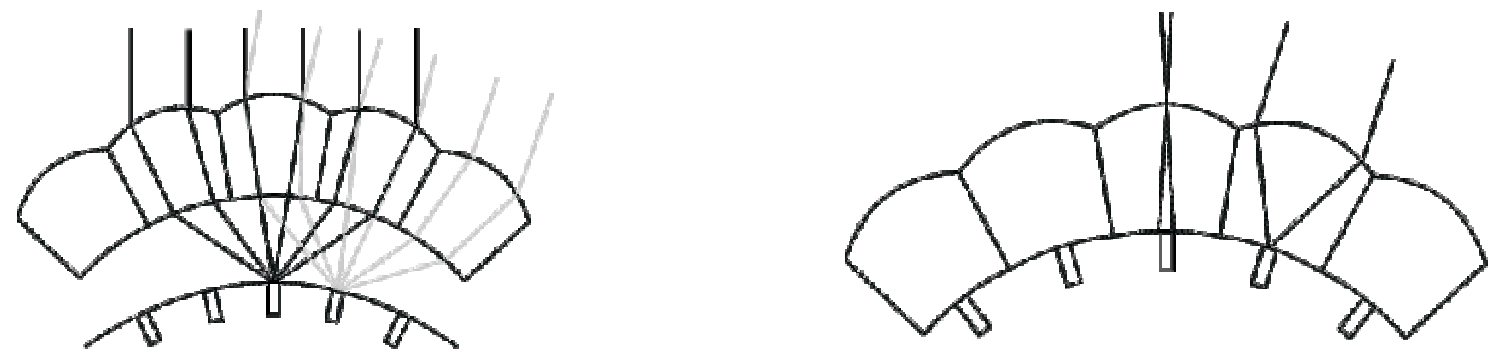

Figure 1. Superposition facet eye (left) and apposition facet eye (right)

In the literature there are already several systems described that perform image transfer through separated optical channels of which some are inspired by nature's insect facet eyes ${ }^{6-8}$ Two of these artificial insect facet eyes are fabricated with wafer-scale techniques: the cluster eye and the artificial apposition compound eye (APCO) ${ }^{6}$ The cluster eye is inspired by the apposition eye and consists of three micro-lens arrays with different pitch. The optical axes of the different channels are thus tilted with respect to each other. Each of these channels samples a small part of the object and makes an intermediate image on the second lens array. On the detector these intermediate images are then brought together to form one single image. The trade-off between resolution and sensitivity is in this case made by the number of lenses in each micro-lens array. The second system, the APCO, is based on the apposition compound eye, where each channel samples one direction in space. It consists of a microlens array with at the focal length of the lenses, just in front of the detector, a pinhole array with slightly different pitch than the microlens array. The optical axes of the system are pointed outwards. Each of these lenses is thus capturing light with a certain angle of incidence. Every micro-lens is optimized for reduction of aberrations under oblique incidence. ${ }^{9}$ Here, the pinhole diameter defines the sensitivity/resolution trade-off. The main restriction of both systems is the FOV because the lenses are not tilted outwards, as is the case in insect facet eyes.

A major diference between the insect facet eyes and the artificial eyes is the shape of the detector which is flat in the artificial case and curved for the insect eyes. From a technological point of view it is more difficult to work with a curved CMOS than with a flat one, although progress has been made in this field. ${ }^{10}$ Because of low-cost and robustness we opted to work with a flat CMOS detector without neglecting the goal to have a good image quality and large FOV.

In the design we made, we want to enlarge the FOV of the system with respect to the cluster eye and APCO and find a good resolution/sensitivity trade-off. For the cluster eye and the APCO, the resolution is $3.3 \mathrm{LP} /{ }^{\circ}$ over a FOV of $33^{\circ}$ by $12^{\circ}$ and $1.6 \mathrm{LP} /{ }^{\circ}$ over a FOV of $25^{\circ}$ by $25^{\circ}$ respectively. In our design we want to enlarge this FOV up to $124^{\circ}$ and reach a resolution below $1^{\circ}$. Our design consists of multiple two micro-lens channels, which are tilted with respect to each other and the optical axis to increase the total FOV and to monitor a good resolution/sensitivity trade-off. We chose to use two micro-lenses per channel since this increases the reduction of aberrations and facilitates the alignment. Because the image plane where a CMOS detector will be positioned captures incident light with an angular difference of $1^{\circ}$, we had to find a good transformation between the incident angles and position of the respective light spots on the image plane. ${ }^{11}$ 


\section{DESIGN OF THE MICRO-OPTICAL SYSTEM}

\subsection{Design Method}

By using 25 separated imaging channels, we want to image the total FOV (which is circular) onto a flat, square detector (which is a square). The detector was divided into 25 zones. There are two spherical coordinates that describe each point in the far field of the image sensor: the angles phi $(\phi)$ and theta $(\theta)$. Light with a certain predefined angle of incidence is imaged in the center of a certain zone of the CMOS detector. By mapping the desired FOV on circles with a radius equal to the parameter $\phi$, we define these center angles of incidence. For a quarter of the total FOV, we define the center directions by varying $\theta$ in three steps for $\phi=25^{\circ}$ and in five steps for $\phi=50^{\circ}$ (Fig. 2).

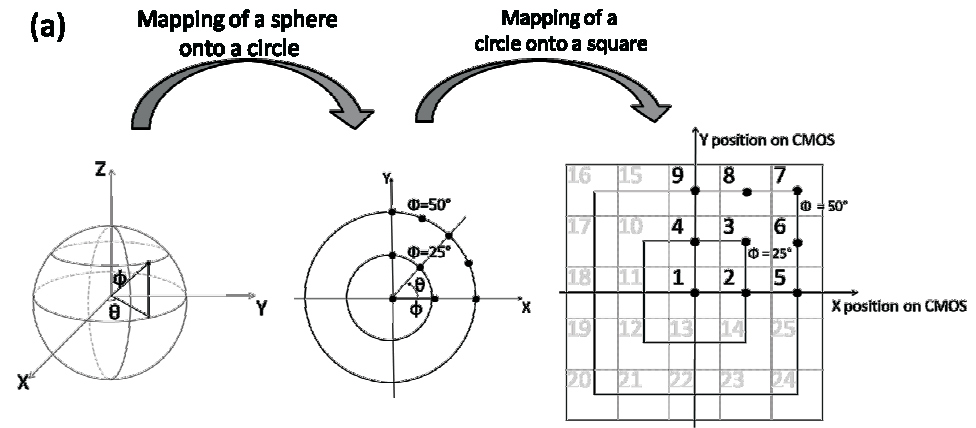

(b)

\begin{tabular}{|l|l|l|l|l|l|l|l|l|l|}
\hline Channel & 1 & 2 & 3 & 4 & 5 & 6 & 7 & 8 & 9 \\
\hline Phl (') & 0 & 25 & 25 & 25 & 50 & 50 & 50 & 50 & 50 \\
\hline Theta (') & 0 & 0 & 45 & 90 & 0 & 22.5 & 45 & 67.5 & 90 \\
\hline
\end{tabular}

Figure 2. Angle To Position Transformation: (a) Mapping of a sphere onto a square. (b) Angles phi $(\phi)$ and theta $(\theta)$ for the center directions of angles of incidence for the first nine channels.

Our goal is to design an optical system that maps these center directions onto the corresponding zones at the detector plane. To sample the complete FOV of $124^{\circ}$, we allow an overlap of the different angular regions mapped by the separate optical channels. Fig. 3 shows a quarter of this total FOV.

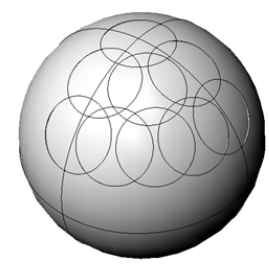

Figure 3. Overlap of the different sub - FOVs.

We can reconstruct a simple image from the different distorted image regions because there is a direct relation between angle of incidence and position on the detector. Our primary design goal is to image the center directions in the center of the CMOS zones and that light with angles of incidence with an angular difference of $1^{\circ}$ creates point spread functions (PSFs) on the detector that do not overlap.

We have 25 channels, each containing two lenses tilted with respect to each other and the optical axis. The lenses, substrates and pedestals will be fabricated in poly-methyl-methacrylate (pmma) material. Fig. 4 shows channel two together with the rays in the center and at the edges of the desired FOV, traced through the optical system. Tab. 1 gives the dimensions of this channel. There are four free parameters for each lens in a single channel: the radius of curvature $(\mathrm{R})$, the conical factor $(\mathrm{C})$ and the tilt of the lens with respect to the $\mathrm{X}\left(T_{X}\right)$ and $\mathrm{Y}\left(T_{Y}\right)$ axis. The function of the first lens is to capture the correct part of light from the total FOV and to 
Table 1. Dimensions of channel two

\begin{tabular}{|c|c|c|}
\hline & Entrance array & Exit array \\
\hline Diameter lens $(\mu \mathrm{m})$ & 250 & 600 \\
\hline Radius of Curvature lens $(\mathrm{mm})$ & -1.39 & 0.68 \\
\hline Tilt of lens around X axis $(\circ)$ & 0 & 0 \\
\hline Tilt of lens around Y axis $(\circ)$ & -22.5 & 11.9 \\
\hline Diameter pedestal on substrate $(\mu \mathrm{m})$ & 500 & 670 \\
\hline Height pedestals $(\mu \mathrm{m})$ & 200 & 130 \\
\hline Thickness substrate $(\mu \mathrm{m})$ & 300 & 350 \\
\hline \multicolumn{2}{|c|}{ Distance between the two lenses: $1000 \mu \mathrm{m}$} \\
\hline
\end{tabular}

have already some focusing towards the second lens. The second lens then focuses the light towards a spot on the detector.

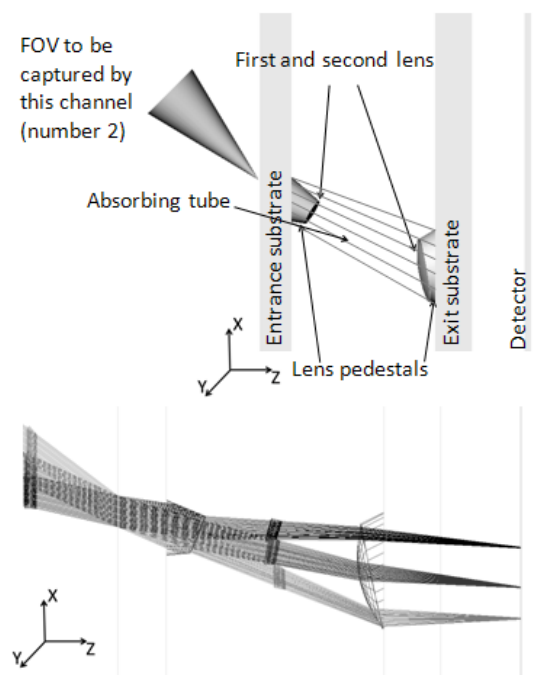

Figure 4. A single channel. Top: schematic with two lenses on a pedestal, tilted with respect to each other and the optical axis. Bottom: Tracing of the rays in the center and at the edge of the sub - FOV through the lenses.

For both lenses the free parameters were optimized using the following figure of merit. We choose three incident beams: the central direction and two at the edge of the FOV of the considered channel. The sum of the diameters of the PSFs originating from these incident beams is then minimized. The design approach to use tilted micro-lenses improves the modulation transfer function (MTF) because it allows to reduce astigmatism, field curvature and distortion, which are severe for large angles of incidence. To prevent stray-light on the detector, we have to isolate the different optical channels from each other. For this reason, we placed the lenses on pedestals and pointed them towards each other. The second lens functions as the field stop of the optical channel. The system is designed such that light with angles of incidence outside the channel's FOV will not pass through the second lens. To prevent light from outside the sub - FOV to reach the detector, we introduce absorbing tubes connecting the first and second lens of each channel (Fig. 5).

\subsection{Obtained image quality}

In Fig. 6, the final design result illustrating the first and second lens array and absorbing tubes in between is shown.

To investigate the optical quality of the complete system, we modeled it in ASAP, ${ }^{12}$ a non-sequential ray tracing software. Optimization of the optical system was also done in ASAP. 


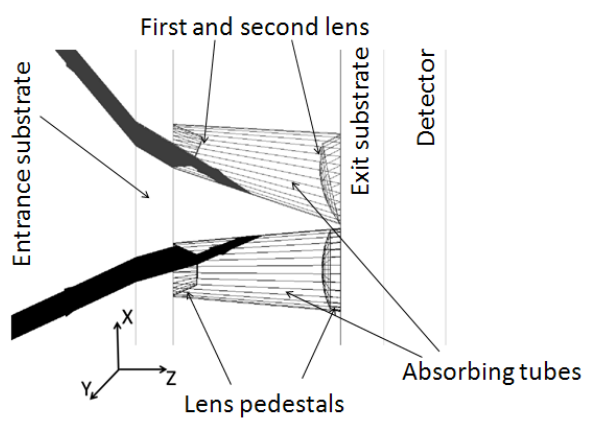

Figure 5. Introducing absorbing tubes to reduce straylight

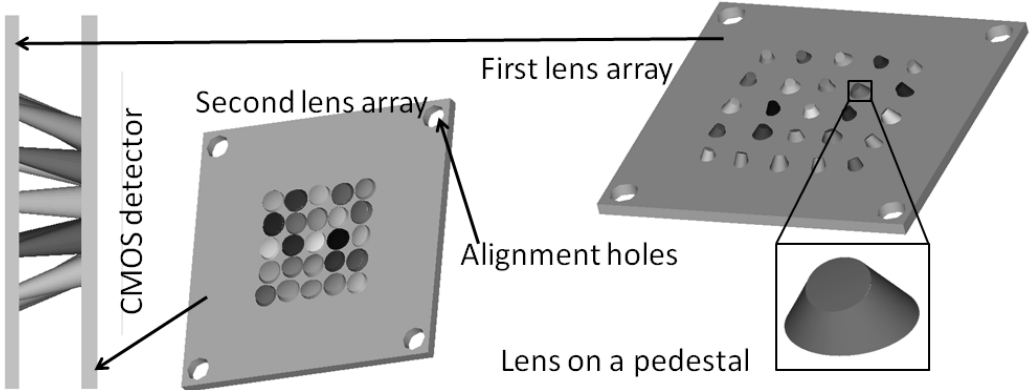

Figure 6. 25 zones of the micro-optical design with side view, complete first and second lens array, absorbing tubes and lens on a pedestal

We define the angular resolution as the smallest angular difference between two incident beams which results in non-overlapping PSFs. We sent light beams with an angular difference of $1^{\circ}$ through the system to check this angular resolution and found that for each of the channels there was no overlap of the PSFs. For channels one, two, four and seven (Fig. 2), we could even do better and reach an angular resolution of $0.5^{\circ}$. Fig. 7 shows the light spots on the detector for channel seven. The central spot and the outer spots originating from the incident rays for which the channel was optimized, are shown. The other spots come from incident rays with an angular difference of $1^{\circ}$. It is clear that all the spots are distinguishable on the detector.

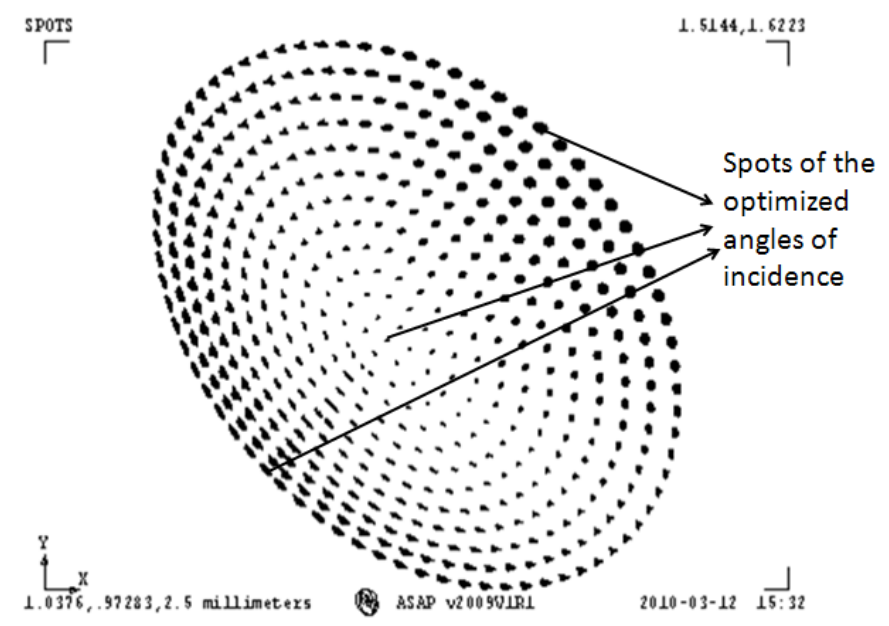

Figure 7. Channel seven: Light spots on the detector. There is an angular resolution of $1^{\circ}$ 
The contrast of the image is a more quantitative figure of merit for the image quality. Therefore we simulated the modulation transfer function (MTF). The result in Fig. 8 shows that up to $0.3 \mathrm{LP} /{ }^{\circ}$ maximum contrast is reached. We attempted to increase the optical throughput of the total system by capturing a relative large FOV for each optical channel. We simulated that $0.3 \%$ of the incident light is arriving at the detector plane. Increasing the diameter of the first lens would increase the optical throughput, but decrease the resolution. The same holds when we would reduce the total number of optical channels and increase the FOV for each individual channel.

\section{Modulation Transfer Function}

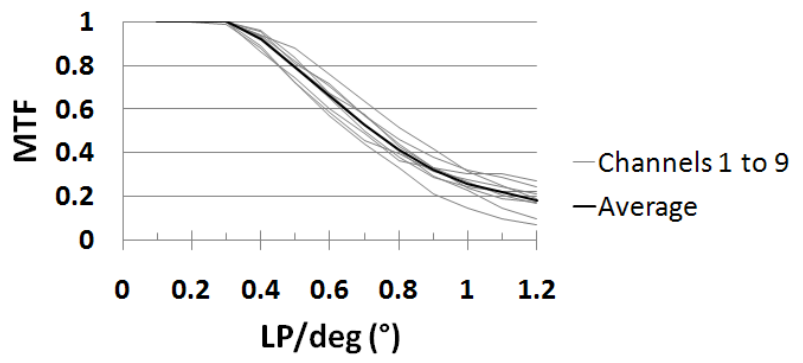

Figure 8. MTF for the different channels of the Double Tilted Lens Design

Each channel is designed to capture light from a certain FOV. In the ideal case, these sub - FOVs would together cover the complete FOV without too much overlap. It is very difficult to avoid overlap when we want to cover the complete FOV due to the problem of mapping a spherical angular distribution to a square detector. This means that certain directions will be sampled by more than one pixel of the CMOS detector. Fig. 9 (left) shows the energy distribution on the detector for channels one to nine. We see that the energy distributions obtained by the different optical channels are well separated which shows that there is no stray-light. Furthermore we see that the energy is decreasing towards the outer region of the sub - FOVs due to vignetting. The lower sensitivity for these directions is solved because it are exactly these directions which are sampled by multiple pixels. Fig. 9 (right) shows that increasing the FOV that is sent through each channel causes some overlap between the energy distributions.
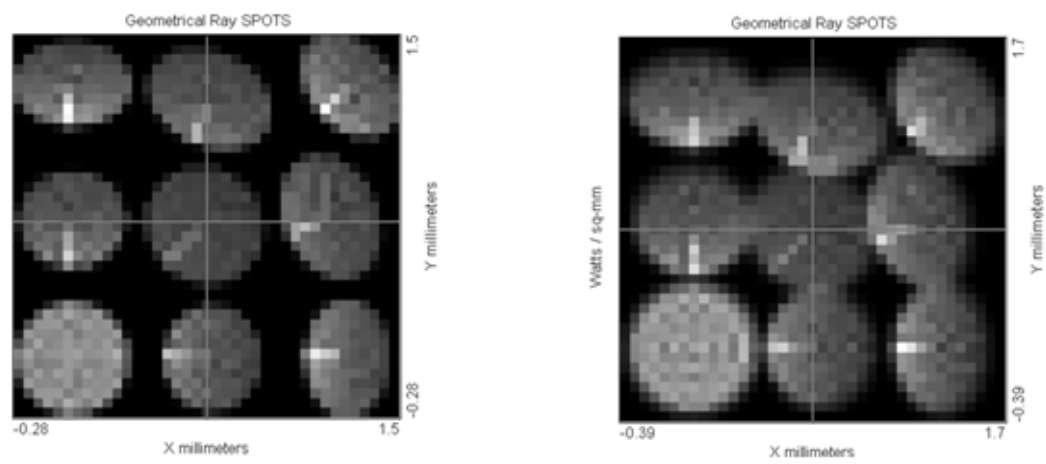

Figure 9. Energy distributions for channels one to nine. Left: Only the FOV for which the channel was designed is sent through the system. Right: increased FOVs are sent through the system; there is some overlap between the energy distributions.

A final parameter to define the quality of the system is distortion, which is different for every channel. Fig.10 shows the effect of distortion. A cross-shaped source was imaged through each channel. Every channel deforms this cross in a slightly different way. In channel seven we find the largest distortion, which is equal to $21 \%$. Since we know for each position on the detector from which angle of incidence the light is coming, we can reconstruct the image by use of image reconstruction algorithms. 


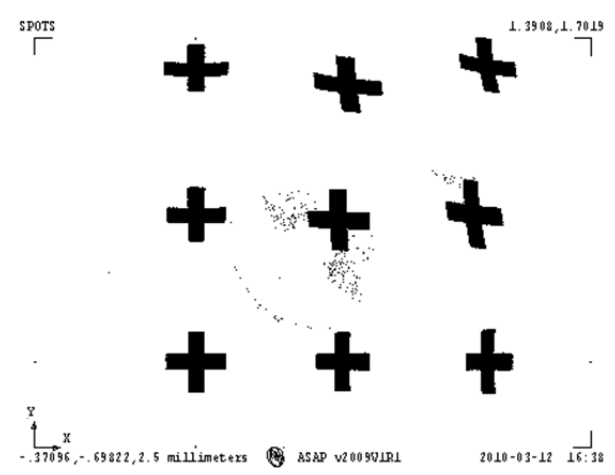

Figure 10. Distortion of cross-shaped sources sent through channels one to nine of the micro-optical system

\section{CONCLUSIONS}

We designed a miniaturized imaging system with a large FOV $\left(124^{\circ}\right)$ and an angular resolution below $1^{\circ}$. The sensitivity of the system is $0.3 \%$ and the maximum distortion is $21 \%$. The MTF shows that maximum contrast in the image is reached up to $0.3 \mathrm{LP} /{ }^{\circ}$. Our designed system is currently being fabricated using state-of-the-art single point diamond turning method. The different components will be aligned on top of a CMOS detector to compare the obtained experimental results with our design results.

\section{ACKNOWLEDGMENTS}

This work was supported in part by the DWTC-IAP, in part by the FWO, in part by the GOA, in part by the 6th FP European Network of Excellence on Micro-Optics(NEMO), in part by the Methusalem and Hercules foundations and in part by the OZR of the Vrije Universiteit Brussel. The work of H. Ottevaere was supported by the Flemish Fund for Scientific Research (FWO) which provided her a "Postdoctoraal Onderzoeker" fellowship.

\section{REFERENCES}

[1] Yamaguchi, T., Nakamoto, M., Sato, Y., Hashizume, M., Sugano, N., Yoshikawa, H., Tamura, S., "Development of camera model and calibration procedure for oblique-viewing endoscope," Transactions of the Institute of Electronics, Information and Communication Engineers D-II J87D-II(1), 313-324 (2004 ).

[2] Yingke, G., Xiang, X., Ziqiang, W., Guolin, L., Tianjia, S., Nan, Q., Chun, Z., Zhihua, W., "A new globularity capsule endoscopy system with multi-camera," IEEE Biomedical Circuits and Systems Conference, 289-292 (2009)

[3] Feng, G., Tian, W., Huang, C., Liu, T., Zhang, S., "Wide field of view CCD camera based on multi-sensors image mosaics," Proc. First International Congress on Image and Signal Processing 2, 432-435 (2008)

[4] Land, M.F. and Nilsson, D.E., Animal Eyes, Oxford Animal Biology Series, Oxford University Press, Oxford, 122-177 (2002).

[5] Völkel, R., Eisner M., Weible, K.J., "Miniaturized imaging systems," Microelectronic Engineering 67-68, 461-472(2003).

[6] Duparré, J., Schreiber, P., Dannberg, P., Scharf, T., Pelli, P., Völkel, R., Herzig, H-P., Brauer, A., "Artificial compound eyes - different concepts and their application to ultra flat image acquisition sensors," Proc. SPIE $5346,89-100$ (2004).

[7] Hornsey, R., Thomas, P., Wong, W., Pepic, S., Yip, K., Krishnasamy, R., "Electronic Compound-Eye Image Sensor: Construction and Calibration," Proc. SPIE 5301,13-24 (2004).

[8] Hamanaka, K., Koshi, H., "An artificial compound eye using a microlens array and its application to scaleinvariant processing," Opt. Rev. 3, 264268 (1996)

[9] Duparré, J., Schreiber, P., Völkel, R., "Theoretical analysis of an artificial superposition compound eye for application in ultra flat digital image acquisition devices," Proc. SPIE 5249, 408-418 (2004). 
[10] Cho Ko,H., Stoykovic, M. P., Song, J., Malyarchuk, V., Mook Choi, W., Yu, C., Geddes III, J. B., Xiao, J.,Wang, S., Huang, Y., Rogers, J., "A hemispherical electronic eye camera based on compressible silicon optoelectronics," Nature 454, 748-753 (2008).

[11] Dresel, T., Beyerlein, M., Schwider, J., "Design of computer-generated beam-shaping holograms by iterative finite-element mesh adaption," Applied Optics 35 (35), 6865-6874 (1996).

[12] http://www.breault.com 